\title{
CAVEATS AND PERSPECTIVES OF COMPUTATIONAL PHOSPHOROUS NMR: SPIN- SPIN COUPLING CONSTANTS
}

Abstract. The second part of two interrelated reviews deals with the computation of ${ }^{31} P \mathrm{NMR}$ spin-spin coupling constants in the diverse phosphorous containing compounds concentrating on their stereochemical dependencies and stereodynamic behavior in the particular classes of organophosphorus compounds.

Keywords: chemical shifts, spin-spin coupling constants, dihedral angle.

\section{Introduction}

Calculation of NMR parameters in a wide variety of chemical compounds and biochemical species has become very popular in the past 10 15 years providing a breakthrough into theoretical and stereochemical aspects of chemical struc-

ture. $^{[1,2,3,4,5,6,7,8,9,10,11,12,13,14,15,16,17,18,19,20,21,22,23,24,25 \text {, }}$

${ }^{26]}$ In particular, calculation of ${ }^{31} \mathrm{P}$ NMR chemical shifts and heteronuclear spin-spin coupling constants involving phosphorous in a wide series of different organophosphorus compounds and phosphorus-containing complexes and clusters together with different bioorganic phosphorus compounds like phosphorus containing nucleosides and nucleotides, natural and synthetic peptides, DNA and RNA is of utmost importance in view of the structural information that could be gained from these calculations.

Computation of spin-spin coupling constants involving phosphorus is much less commonly undertaken compared to those of ${ }^{31} \mathrm{P}$ NMR chemical shifts, primarily due to the strong dihedral angle dependencies of these parameters requiring exact knowledge of the stereochemistry of the compounds under study, which is often not so obvious and/or unequivocal. Despite the challenges, such calculations do exist and are briefly outlined below, focusing primarily on the computational aspects of spinspin coupling constants involving phosphorus nucleus in particular classes of organophosphorus compounds.

\section{Spin-spin coupling constants ${ }^{31} \mathbf{P}-{ }^{1} \mathrm{H}$ and ${ }^{31} \mathrm{P}$ - ${ }^{13} \mathbf{C}$}

The flow of papers dealing with the computation of spin-spin coupling constants involving phosphorus is more sparse compared to stud- ies focused on the calculation of ${ }^{31} \mathrm{P}$ NMR chemical shifts. However, the former do exist, and in this review, we shall briefly concentrate on the computational aspects of mostly ${ }^{31} \mathrm{P}-{ }^{1} \mathrm{H}$ and ${ }^{31} \mathrm{P}-{ }^{13} \mathrm{C}$ couplings leaving other exotic types of spin-spin coupling constants including phosphorus nucleus for a more comprehensive survey.

The story begins with a most simple phosphine, $\mathrm{PH}_{3}$, thoroughly investigated by Garbacz, Makulski, and Jaszuński ${ }^{[27]}$ who performed a state-of-the-art calculations of its ${ }^{1} J_{\mathrm{PH}}$ spin-spin coupling constant in comparison with a gas-phase experimental data. The line shape analysis of ${ }^{1} \mathrm{H}$ and ${ }^{31} \mathrm{P}$ gas-phase NMR spectra were recorded at several densities of $\mathrm{PH}_{3}$, followed by the extrapolation of the results to a zero-density limit, which gave $176.18 \mathrm{~Hz}$ as the experimental value of this coupling at $300 \mathrm{~K}$. On the other hand, the most sophisticated CCSD calculations in combination with the penta-zeta quality basis sets of Dunning and Jensen gave $187.86 \mathrm{~Hz}$ as the nonrelativistic equilibrium geometry value, while adding relativistic and temperature corrections resulted in $177.14 \mathrm{~Hz}$ at 300 $\mathrm{K}$, in a notably good agreement with experiment $(176.18 \mathrm{~Hz})$. It appeared that agreement between the experimental and computed spin-spin coupling in the $\mathrm{PH}_{3}$ molecule was obtained only when nuclear relaxation and intermolecular interactions were taken into account. It was thus demonstrated that negligibly small discrepancy between CCSD calculations and experiment was due to the neglect of these two effects.

In a much earlier publication by Wrackmeyer, ${ }^{[28]}$ calculation of spin-spin coupling constants ${ }^{1} J_{\mathrm{PC}}$ across triple $\mathrm{P} \equiv \mathrm{C}$ bond of $\lambda^{3}$-1-phosphaalkynes, $\mathrm{P} \equiv \mathrm{C}-\mathrm{R}(\mathrm{R}=\mathrm{H}, \mathrm{Me}, t$-Bu, $\mathrm{Ph}, \mathrm{SiMe}_{3}$, and $\mathrm{NMe}_{2}$ ), at the DFT B3LYP/6- 
$311+\mathrm{G}(\mathrm{d}, \mathrm{p})$ level of theory have revealed a positive sign of this coupling constant, in agreement with the available experimental value for a $t-\mathrm{Bu}-$ $\mathrm{C} \equiv \mathrm{P}$ molecule. Calculations performed showed that the Fermi contact contribution to this coupling was unexpectedly negative, and that positive sign of the total coupling, ${ }^{1} J_{\mathrm{PC}}$, was the result of the significant contributions arising from the spin-dipole and paramagnetic spin-orbital terms.

In two related publications by Fedorov, et $a l .^{[29,30]}$ dealing with the calculation of geminal and vicinal phosphorus-proton coupling constants, ${ }^{2} J_{\mathrm{PH}}$ and ${ }^{3} J_{\mathrm{PH}}$, in trivinyl phosphine and trivinyl phosphine chalcogenides, it was found that each of the four compounds of this series existed in the equilibrium mixture of five trueminimum conformers, namely s-cis-s-cis-s-cis, s-cis-s-cis-gauche, syn-s-cis-gauche-gauche, anti-s-cis-gauche-gauche, and gauche-gauchegauche. Results of the theoretical energy-based conformational analyses performed were generally in agreement with the earlier theoretical and experimental data for the related species, unsaturated phosphines and phosphine chalcogenides, demonstrating predominance of the planar s-cis conformer in all cases, especially in phosphine chalcogenides, as compared to phosphines.

The most interesting result, reported in those communications, ${ }^{[29,30]}$ was that the ${ }^{2} J_{\mathrm{PH}}$ and ${ }^{3} J_{\mathrm{PH}}$ coupling constants provided marked stereospecificity with respect to the orientation of the phosphorus lone pair and that of the $\mathrm{P}=\mathrm{X}$ double bonds $(\mathrm{X}=\mathrm{O}, \mathrm{S}, \mathrm{Se})$, as exemplified for trivinylphosphine in Figure 1. It is seen that both vicinal couplings, ${ }^{3} J\left(\mathrm{P}, \mathrm{H}_{\mathrm{A}}\right)$ and ${ }^{3} J\left(\mathrm{P}, \mathrm{H}_{\mathrm{B}}\right)$, decreased while the geminal coupling, ${ }^{2} J\left(\mathrm{P}, \mathrm{H}_{\mathrm{X}}\right)$, increased in going from the cisoidal to the transoidal orientation of the rotating vinyl group with respect to the phosphorus lone pair. What is most exciting, is that geminal coupling, ${ }^{2} J\left(\mathrm{P}, \mathrm{H}_{\mathrm{X}}\right)$, increased by as much as $c a .70 \mathrm{~Hz}$ which is, indeed, a very strong conformational effect. It is noteworthy that cisoidal, ${ }^{3} J\left(\mathrm{P}, \mathrm{H}_{\mathrm{B}}\right)$, and transoidal, ${ }^{3} J\left(\mathrm{P}, \mathrm{H}_{\mathrm{A}}\right)$, vicinal couplings differ considerably (by $c a .20$ and $40 \mathrm{~Hz}$, respectively) in the cisoidal orientation of the vinyl group $(\phi$ $=0^{0}$ ), while they are almost equal $(\mathrm{ca} 14-15 \mathrm{~Hz})$ in the transoidal conformation $\left(\phi=180^{\circ}\right)$. The latter observation is a strong indication of a possible caveat dealing with the configurational assignment at the double bond in the unsaturated phosphines based on vicinal phosphorushydrogen coupling constants. This finding implied that a special care should be taken in stereochemical studies of unsaturated phosphines and phosphine chalcogenides based on the phosphorus-proton spin-spin coupling constants. To avoid misleading conclusions and erroneous spectral assignments based on ${ }^{2} J_{\mathrm{PH}}$ and ${ }^{3} J_{\mathrm{PH}}$ coupling constants, the established trends of their stereochemical behavior reported in those papers ${ }^{[29,30]}$ should first be taken into account before any of their conformational applications are in progress.

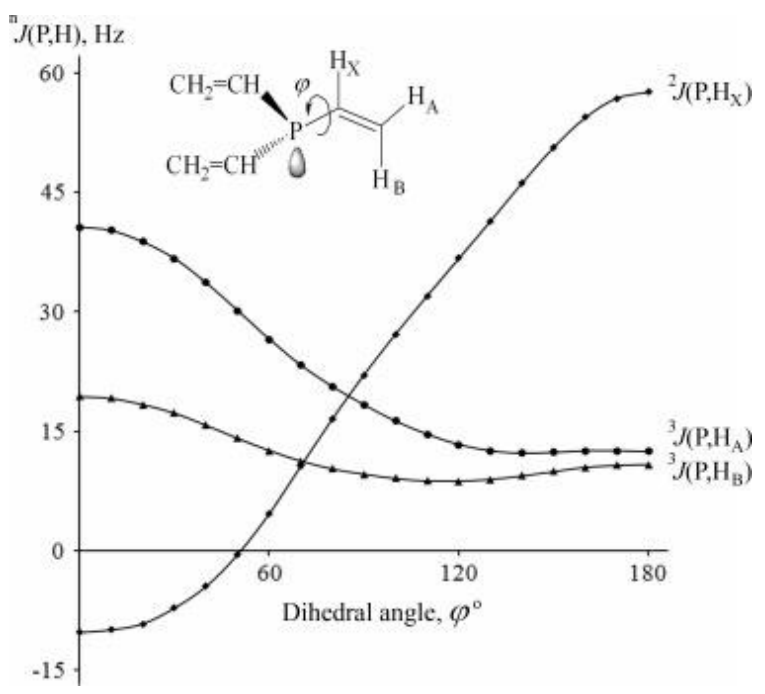

Figure 1. Dihedral angle dependencies of ${ }^{2} J_{\mathrm{PH}}$ and ${ }^{3} J_{\mathrm{PH}}$ couplings in trivinylphosphine calculated at the SOPPA/aug-cc-pVTZ-J level. The value of $\phi=0^{0}$ is assigned to the $s$-cis orientation of the vinyl group and phosphorus lone pair. Reproduced from Fedorov, et al. ${ }^{[29]}$ with the permission of John Wiley and Sons. 
Very recently, Rusakov et $a l^{[31]}$ performed a state-of-the-art SOPPA(MP2) calculation of ${ }^{2} J_{\mathrm{PC}}$ and ${ }^{3} J_{\mathrm{PC}}$ coupling constants in the series of four heterocyclic phosphines that were compared with experimental data ${ }^{[32]}$ taking into account their stereochemical dependencies and considering relativistic and solvent effects. Solvent corrections were calculated as the differences between their values obtained in liquid and gas phases within the IEF-PCM scheme at the DFT level while relativistic corrections were evaluated at the four-component level within the unrestricted kinetic balance scheme ${ }^{[33]}$ the latter for the sake of avoiding the variational collapse problem. ${ }^{[34,35]}$

A number of the locally dense basis set schemes were thoroughly tested in this study in terms of their accuracy versus computational demands. The most efficient scheme implied Jensen's basis sets of quadruple zeta quality augmented with diffuse functions on phosphorus and carbons, aug-pcS-3, and Dunning's triple zeta quality diffuse aug-cc-pVTZ basis set on the nearest carbon atoms together with a double zeta cc-pVDZ basis set on the rest of atoms. It was shown that suggested computational scheme provided a very good correlation between ${ }^{2} J_{\mathrm{PC}}$ calculated at the SOPPA(MP2) level and experiment with a mean absolute error of about 1.5 $\mathrm{Hz}$ in the range of about $30 \mathrm{~Hz}$.
A marked dihedral angle dependence of ${ }^{2} J_{\mathrm{PC}}$ was demonstrated in that paper, as shown in Figures 2 and 3, which could be useful in stereochemical studies of a wide series of organophosphorus compounds. The strong orientational effect of the phosphorus lone pair on ${ }^{2} J_{\mathrm{PC}}$ is well known. ${ }^{[36,37]}$ However, in that paper, ${ }^{[31]}$ this effect was reestablished at the high-accuracy correlated SOPPA(MP2) level in combination with the four-component DFT relativistic corrections, in contrast to the previous studies, performed within the nonrelativistic DFT approach. As one can see in Figures 2 and $3,{ }^{2} J_{\mathrm{PC}}$ manifests a marked stereospecificity in both cases, which could be useful in stereochemical studies of a wide series of organophosphorus compounds. It was found that the values of ${ }^{2} J_{\mathrm{PC}}$ varied in a wide range of about $40-50 \mathrm{~Hz}$. As followed from the calculations performed, this profound stereochemical behavior of ${ }^{2} J_{\mathrm{PC}}$ was totally determined by the Fermi-contact contribution in both cases. In contrast, stereochemical dependencies of the onebond ${ }^{31} \mathrm{P}-{ }^{13} \mathrm{C}$ coupling constants were found to be much less expressive. Moreover, the noncontact contributions of ${ }^{1} J_{\mathrm{PC}}$ were found to be essential due to the mutual cancellation of the Fermi-contact and the non-contact contributions.

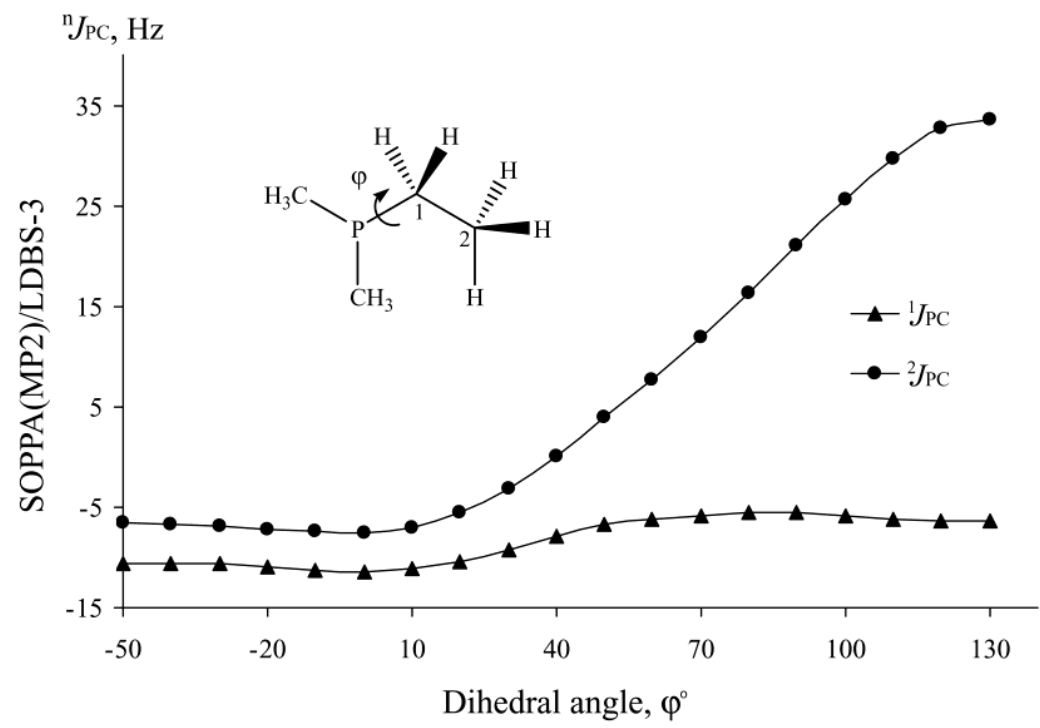

Figure 2. Dihedral angle dependence of ${ }^{1} J_{\mathrm{PC}}$ and ${ }^{2} J_{\mathrm{PC}}$ in ethyl(dimethyl)phosphine. The value of $\varphi$ $=-50^{\circ}$ corresponds to the antiperiplanar orientation of the carbon-carbon bond towards the phosphorus lone pair. Reproduced from Y.Y. Rusakov, et al. ${ }^{[31]}$ with the permission of the American Chemical Society. 


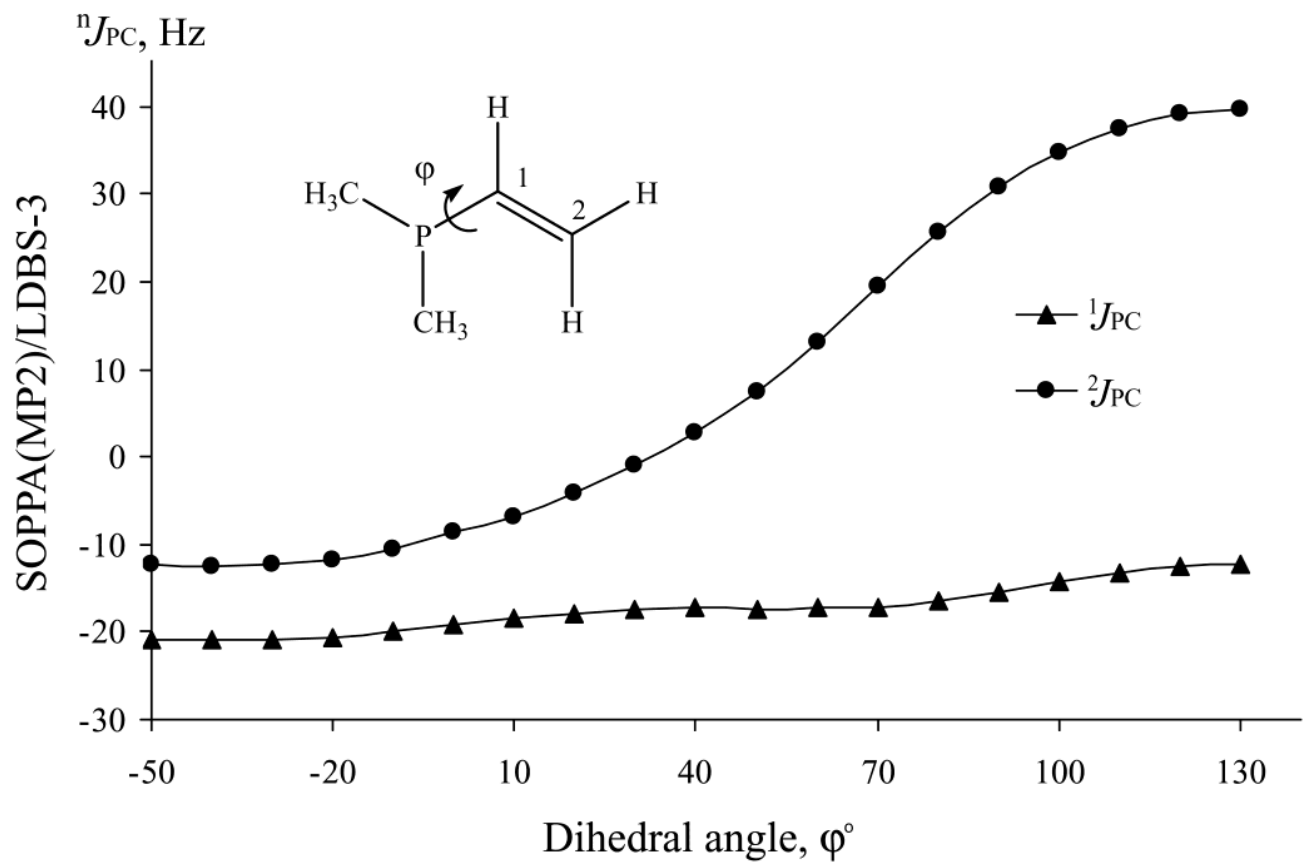

Figure 3. Dihedral angle dependence of ${ }^{1} J_{\mathrm{PC}}$ and ${ }^{2} J_{\mathrm{PC}}$ in vinyl(dimethyl)phosphine. The value of $\varphi=-$ $50^{\circ}$ corresponds to the antiperiplanar orientation of the carbon- carbon bond towards the phosphorus lone pair. Reproduced from Y.Y. Rusakov, et al. ${ }^{[31]}$ with the permission of the American Chemical Society.

Very recently Gholivand, et al. ${ }^{[38]}$ performed DFT calculation of ${ }^{2} J_{\mathrm{PC}}$ coupling constants in the series of phosphoryl benzamides, $\mathrm{C}_{6} \mathrm{H}_{5} \mathrm{C}(\mathrm{O}) \mathrm{NHP}(\mathrm{O}) \mathrm{R}^{1} \mathrm{R}^{2}$, with $\mathrm{R}^{1}$ and $\mathrm{R}^{2}$ being aziridine, azetidine, pyrrolidine, piperidine, azepane, 4-methylpiperidine, propane-2-amine, and 2-methylpropane-2-amine. Calculated ${ }^{2} J_{\mathrm{PC}}$ couplings were in a reasonably good agreement with available experimental data, and their substituent trends were interpreted in terms of geometric and electronic properties, the latter involving atomic charge and hybridization effects on phosphorous.

As an example of the biochemical applications of computational spin-spin coupling constants involving phosphorous, Sychrovský, et $a l .{ }^{[39,40]}$ performed B3LYP/IGLO-II(III) calculations of ${ }^{\mathrm{n}} J_{\mathrm{PH}}$ and ${ }^{\mathrm{n}} J_{\mathrm{PC}}(\mathrm{n}=2,3,4)$ in the model fragments across the P-O $\cdots \mathrm{H}-\mathrm{C}$ linkage between the nucleic acid backbone phosphate and the nucleic acid base used for the structure determination of nucleic acids. It was shown that ${ }^{3} J_{\mathrm{PC}}$ and ${ }^{3} J_{\mathrm{PH}}$ demonstrated a pronounced Karplus-type dihedral angle dependence, as illustrated for the model complex of guanine base interacting with dimethyl-phosphate $(\mathrm{G} \cdots \mathrm{dmP})$. These results provided application of ${ }^{3} J_{\mathrm{PC}}$ and
${ }^{3} J_{\mathrm{PH}}$ in stereochemical analysis of the phosphorus containing DNA base pairs and related complexes.

In the forthcoming paper by the same principal authors, ${ }^{[41]}$ the measured coupling constants, ${ }^{\mathrm{n}} J_{\mathrm{PH}}$ and ${ }^{\mathrm{n}} J_{\mathrm{PC}}$, in the RNA dinucleoside monophosphates were assigned to the glycosidic torsion angles in order to resolve the global structure of dinucleoside monophosphates. In this study, experimental coupling constants were correlated with their theoretical values obtained as the dynamical averages of the Karplus equations relevant to the torsion angles.

In a more theoretical paper from the same group ${ }^{[42]}$ the ${ }^{2} J_{\mathrm{P}, \mathrm{C} 3^{\prime}},{ }^{2} J_{\mathrm{P}, \mathrm{C} 5^{\prime}}$, and ${ }^{3} J_{\mathrm{P}, \mathrm{C} 4^{\prime}}$ coupling constants were calculated in dependence on nucleic acid backbone torsion angles $\zeta$ and $\alpha$, as was specified for a parent nucleic acid and a model ethyl methyl phosphate. DFT calculations of ${ }^{2} J_{\mathrm{PC}}$ coupling constants in nucleic acid backbone performed by these authors revealed their strong dependence on both torsion angles, $\zeta$ and $\alpha$, with a dominant modulation by only one of the torsion angles, as illustrated in Figure 4, showing two-dimensional ${ }^{2} J_{\mathrm{PC}}$ surface of $\alpha$ and $\zeta$ torsion angles of the model nucleic acid phosphate. In this way, the ${ }^{2} J_{\mathrm{P}, \mathrm{C} 3^{\prime}}$ and ${ }^{2} \boldsymbol{J}_{\mathrm{P}, \mathrm{C} 5^{\prime}}$ couplings 
were structurally assigned to the torsion angles $\zeta$ and $\alpha$, respectively, and interpreted as the restraints on the phosphate conformation. However, the absolute ${ }^{2} J_{\mathrm{PC}}$ values, calculated with the B3LYP/PCM method, were overestimated overall relative to experiment. Generally, these re-

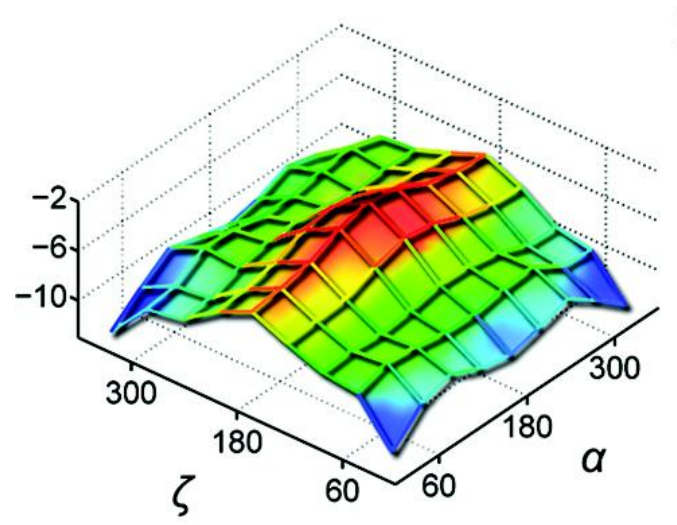

sults provided the missing link between the ${ }^{2} \boldsymbol{J}_{\mathrm{P}, \mathrm{C} 3^{\prime}}$ and ${ }^{2} \boldsymbol{J}_{\mathrm{P}, \mathrm{C} 5^{\prime}}$ spin-spin coupling constants and the nucleic acid backbone torsion angles $\zeta$ and $\alpha$.

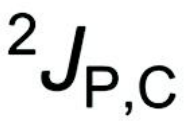

Figure 4. Two-dimensional ${ }^{2} J_{\mathrm{PC}}$ surface of $\alpha$ and $\zeta$ torsion angles of the model nucleic acid phosphate. Reproduced from Benda, et al. ${ }^{[42]}$ with the permission of the American Chemical Society.

Furthermore, it was found that the ${ }^{2} J_{\mathrm{P}, \mathrm{C} 5^{\prime}}$ coupling depended dominantly on the torsion angle $\alpha$ while the ${ }^{2} J_{\mathrm{P}, \mathrm{C} 3^{\prime}}$ coupling strongly depended primarily on the torsion angle $\zeta$. From these constraints, the ${ }^{2} J_{\mathrm{PC}}$ couplings were negative for all phosphate conformations ranging approximately from 3 to $13 \mathrm{~Hz}$ in absolute value, as shown in Figure 5. Based on these dependencies, some conformers of nucleic acid phosphate could be clearly distinguished by means of ${ }^{2} J_{\mathrm{PC}}$ couplings. In general, performed in this study calculations of ${ }^{2} J_{\mathrm{PC}}$ provided an important link between the simple ethyl methyl

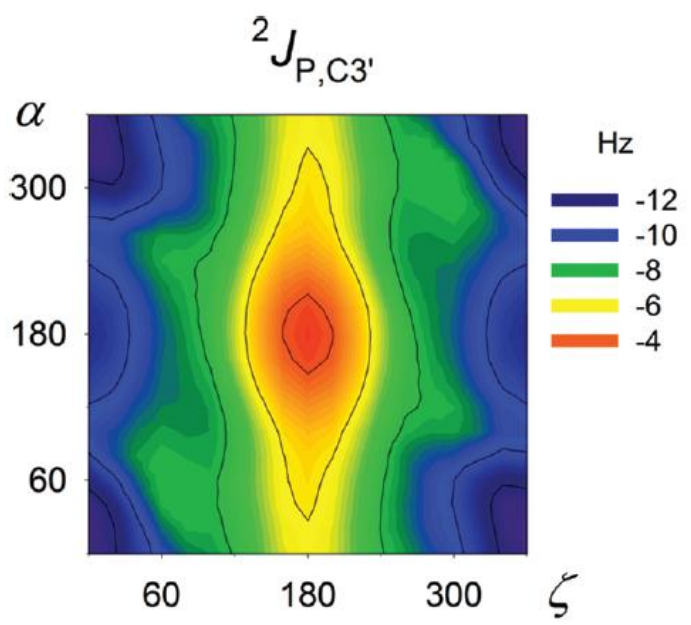

phosphate model and the actual nucleic acid backbone structure.

Structural interpretation of the ${ }^{2} J_{\mathrm{P}, \mathrm{C} 3^{\prime}}$ and ${ }^{2} J_{\mathrm{P}, \mathrm{C} 5^{\prime}}$ couplings proposed by the authors ${ }^{[40]}$ were only qualitative, namely because the twodimensional character of their dependencies implied that similar ${ }^{2} J_{\mathrm{PC}}$ values could correspond to different phosphate conformers. Nevertheless, it was unequivocally found that the ${ }^{2} \boldsymbol{J}_{\mathrm{P}, \mathrm{C} 3^{\prime}}$ and ${ }^{2} J_{\mathrm{P}, \mathrm{C} 5^{\prime}}$ couplings could be definitely assigned to the nucleic acid backbone torsion angles, $\zeta$ and $\alpha$, respectively.

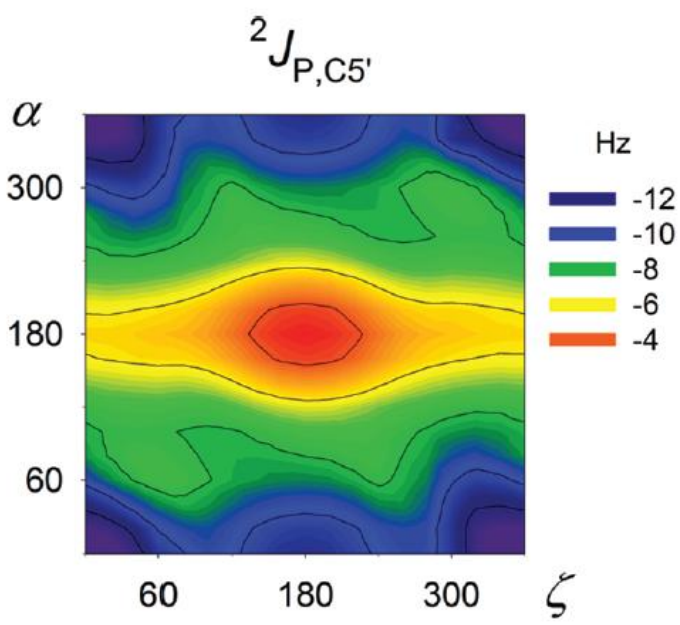

Figure 5. Dependencies of the ${ }^{2} J_{\mathrm{P}, \mathrm{C} 3^{\prime}}$ and ${ }^{2} J_{\mathrm{P}, \mathrm{C} 5^{\prime}}$ coupling constants on the $(\zeta, \alpha)$ conformation of the model ethyl methyl phosphate. Reproduced with minor editing privilege from Benda, et al. ${ }^{[42]}$ with the permission of the American Chemical Society. 
In the following publication by the same principal authors, ${ }^{[43]}$ the dependence of ${ }^{2} J_{\mathrm{PC}}$ coupling constants on solvation of nucleic acid phosphate by $\mathrm{Mg}^{2+}$ and water was thoroughly studied by using the methods of bioinformatic structural analyses of crystallographic data and the DFT calculations of these coupling constants. Calculations performed highlighted the crucial importance of the local $\mathrm{Mg}^{2+}$ coordination geometry, including hydration by explicit water molecules, and necessity of the dynamical averaging over the solvent reorientation in the calculation of phosphorus-carbon coupling constants.

It is well known that nucleic acids demonstrate the self-alignment effects in strong magnetic fields resulting in the appearance of direct spin-spin interactions, known as residual dipolar couplings, revealing the relative orientations of the internuclear vectors with respect to the direction of the external magnetic field. In this line, the direct spin-spin interactions, including those between phosphorus and protons, ${ }^{\mathrm{n}} J_{\mathrm{PH}}(\mathrm{n}=2,3,4)$, were measured under the con- ditions where the studied molecules were partially oriented with respect to the magnetic field. ${ }^{[4]}$ Performed in this paper DFT calculations demonstrated that in some cases these effects could indeed be traced out in the values of ${ }^{\mathrm{n}} \boldsymbol{J}_{\mathrm{PH}}$ desvribing the DNA backbone.

\section{Other spin-spin coupling constants involving phosphorus}

As it has been mentioned earlier, the flow of reports dealing with the computation of spinspin coupling constants involving phosphorus is more sparse when compared to those dealing with the calculation of ${ }^{31} \mathrm{P}$ NMR chemical shifts. This is probably due to the fact that these couplings are much less commonly encountered in chemical practice, as compared to ${ }^{n} J_{\mathrm{PH}}$ and ${ }^{\mathrm{n}} J_{\mathrm{PC}}$.

As an illustrative example of such studies, one-bond phosphorus couplings involving different nuclei, ${ }^{1} J_{\mathrm{PX}}(\mathrm{X}=\mathrm{H}, \mathrm{O}, \mathrm{S}, \mathrm{Se}, \mathrm{C}$ and $\mathrm{N})$, were calculated by Pecul, et al. ${ }^{[45]}$ within the DFT framework with a variety of functionals and basis sets in the series of diverse dioxaphosphorinanes presented in Figure 6.

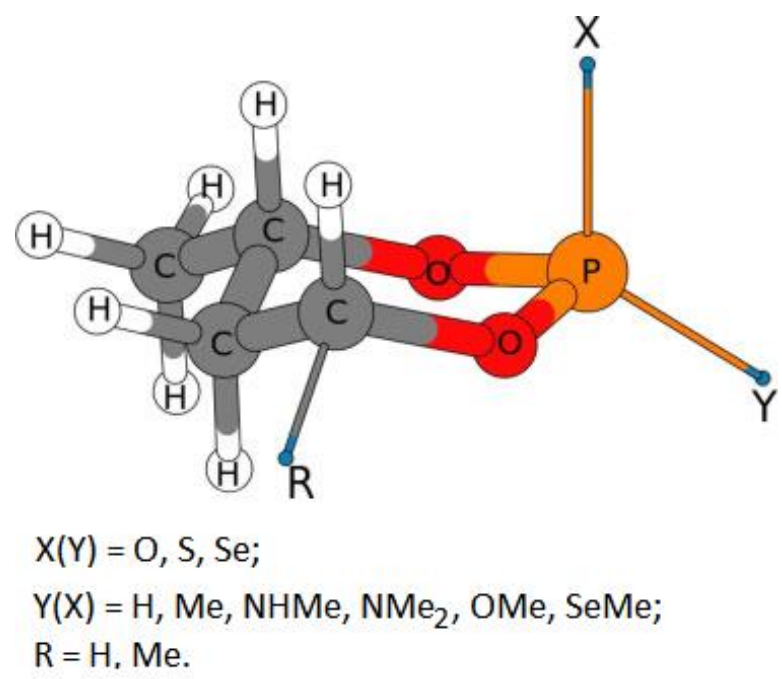

Figure 6. The structures of substituted dioxaphosphorinanes. Reproduced from Pecul, et al ${ }^{[45]}$ with minor editing privilege with the permission of John Wiley and Sons.

It was found by the authors ${ }^{[45]}$ that almost all computed spin-spin coupling constants between phosphorus nucleus and axial and equatorial substituents, involving proton, oxygen, sulfur, selenium, carbon, and nitrogen, were in most cases, essentially overestimated. At the same time, these errors were practically identical in a pair of isomers, and thus canceled each oth- er in the comparison of the coupling with the axial and equatorial substituent. Therefore, the experimentally observed differences between spin-spin coupling constant of phosphorus and axial or equatorial substituents were in each case qualitatively reproduced. This indicated that such experimentally observed trends may be confirmed at the DFT level. It was also found 
that the differences of the coupling constants between phosphorus nucleus and axial and equatorial substituents were correlated with the difference between the respective bond lengths.

\section{Conclusions}

In conclusion it should be noted that a number of papers dealing with computation of spin-spin coupling constants involving phosphorus is less when compared to the attention that has been focused on the calculation of ${ }^{31} \mathrm{P}$ NMR chemical shifts. These papers are concentrated mainly on the calculation of ${ }^{31} \mathrm{P}-{ }^{1} \mathrm{H}$ and ${ }^{31} \mathrm{P}_{-}{ }^{13} \mathrm{C}$ couplings while computation of other exotic types of spin-spin coupling constants including phosphorus nucleus are much more rare and are of almost no practical interest as yet. However, we do hope that this situation will begin to change in the next several years in view of a general marked progress in computational NMR capabilities.

\section{Glossary of abbreviations}

CBS: Complete Basis Set

CCSD: Coupled Cluster Singles and Doubles

$\operatorname{CCSD}(T)$ : Coupled Cluster Singles and Doubles with Perturbative Triples Corrections

CPCM: Conductor-like Polarizable Continuum Model

DFT: Density Functional Theory
HALA: Heavy Atom on Light Atom (effect)

$H F$ : Hartree-Fock

IEF-PCM: Integral Equation Formalism Polarizable Continuum Model

LDBS: Locally Dense Basis Set

$M A D$ : Mean Absolute Deviation

$M A E$ : Mean Absolute Error

MP2: Second-Order Møller-Plesset Perturbation

Theory

NMR: Nuclear Magnetic Resonance

QM/MM: Quantum Mechanics/Molecular Mechanics

$H F$ : Hartree-Fock

$R M S$ : Root Mean Square

ONIOM: Own N-layered Integrated Molecular Orbital Method

SSM: Supermolecular Solvation Model

ZORA: Zero $^{\text {th }}$ Order Regular Approximation

$Z P V C$ : Zero-Point Vibrational Correction

Acknowledgement. The author is grateful to Academician Boris Trofimov and Professor Nina Gusarova (A. E. Favorsky Irkutsk Institute of Chemistry), who have inspired his interest in phosphorus NMR. The invaluable help of Professor Gary Martin (Seton Hall University and Stevens Institute of Technology) in preparing the manuscript of this review for publication is very much appreciated.

\section{References}

[1] S. P. A. Sauer, Molecular Electromagnetism. A Computational Chemistry Approach, University Press, Oxford, 2012.

[2] T. Helgaker, M. Jaszuński, K. Ruud. Chem. Rev. 1999, 99, 293-352.

[3] T. Helgaker, M. Jaszuński, M. Pecul. Prog. NMR Spectrosc. 2008, 53, 249-268.

[4] T. Helgaker, S. Coriani, P. Jørgensen, K. Kristensen, J. Olsen, K. Ruud. Chem. Rev. 2012, 112, 543-631.

[5] D. Cremer, J. Grafenstein. Phys. Chem. Chem. Phys. 2007, 9, 2791-2816.

[6] J. Vaara. Phys. Chem. Chem. Phys. 2007, 9, 5399-5418.

[7] G. A. Aucar, R. H. Romero, A. F. Maldonado. Int. Rev. Phys. Chem. 2010, 29, 164.
[8] M. Bühl, T. van Mourik. WIREs Comput. Mol. Sci. 2011, 1, 634-647.

[9] High Resolution NMR Spectroscopy: Understanding Molecules and their Electronic Structures (Ed: R.H. Contreras), Elsevier, The Netherlands, 2013.

[10] Y. Y. Rusakov, L. B. Krivdin. Russ. Chem. Rev. 2013, 82, 99-130.

[11] F. A. A. Mulder, M. Filatov. Chem. Soc. Rev. 2010, 39, 578-590.

[12] L. B. Krivdin, R. H. Contreras. Ann. Rep. NMR Spectrosc. 2007, 61, 133-245.

[13] L. B. Krivdin, Y. Y. Rusakov. Encycl. Magn. Reson. 2014, 3, 87-110.

[14] A. Navarro-Vázquez. Magn. Reson. Chem. 2017, 55, 29-32.

[15] Y. Xiao, W. Liu, J. Autschbach. Relativistic Theories of NMR Shielding, in: Handbook of Relativistic Quantum 
Chemistry (Ed: W. Liu), Springer, Berlin, 2017.

[16] J. Autschbach. Phyl. Trans. Royal Soc. A 2014, 372, 1-39.

[17] M. Repisky, S. Komorovsky, R. Bast, K. Ruud. Relativistic Calculations of $\mathrm{Nu}$ clear Magnetic Resonance Parameters, in: Gas phase NMR (Eds: K. Jackowski and M. Jaszunski), The Royal Society of Chemistry, Cambridge, 2016.

[18] I. L. Rusakova, Y. Y. Rusakov, L. B. Krivdin. Russ. Chem. Rev. 2016, 85, 356-426.

[19] I. L. Rusakova, L. B. Krivdin. Mendeleev Commun. 2018, 28, 1-13.

[20] L. B. Krivdin, Prog. NMR Spectrosc. 2017, 102-103, 98-119.

[21] L. B. Krivdin, Prog. NMR Spectrosc. 2018, 105, 54-99.

[22] L. B. Krivdin, Prog. NMR Spectrosc. 2018, 108, 17-73.

[23] L. B. Krivdin, Prog. NMR Spectrosc. 2019, 112-113, 103-156.

[24] L. B. Krivdin, Magn. Reson. Chem. 2019, doi $10.1002 / \mathrm{mrc} .4873$

[25] L. B. Krivdin, Magn. Reson. Chem. 2019, doi $10.1002 / \mathrm{mrc} .4896$

[26] L. B. Krivdin, Magn. Reson. Chem. 2019, doi 10.1002/mrc.4895

[27] P. Garbacz, W. Makulski, M. Jaszuński, Phys. Chem. Chem. Phys. 2014, 16, 21559-21563.

[28] B, Wrackmeyer, Z. Naturforsch. 2003, $58 b, 1041-1044$.

[29] S. V. Fedorov, L. B. Krivdin, Y. Y. Rusakov, N. A. Chernysheva, V. L. Mikhailenko, Magn. Reson. Chem. 2010, 48, S48-S55.

[30] S. V. Fedorov, L. B. Krivdin, Y. Y. Rusakov, I. A. Ushakov, N. V. Istomina, N. A. Belogorlova, S. F. Malysheva, N. K. Gusarova, B. A. Trofimov, Magn. Reson. Chem. 2009, 47, 288-299.

[31] Y. Y. Rusakov, I. L. Rusakova, S. V. Fedorov, G. A. Gray, L. B. Krivdin, $J$. Phys. Chem. A 2019, 123, 6298-6303.

[32] G. A. Gray, S. E. Cremer, K. L. Marsi, J. Am. Chem. Soc. 1976, 98, 2109-2118.

[33] U. Kaldor, S. Wilson, Theoretical Chemistry and Physics of Heavy and Super- heavy Elements, SpringerScience+Business Media, B. V., Dordrecht, 2003, $565 \mathrm{p}$.

[34] W. H. E. Schwarz, H. Wallmeier, Mol. Phys. 1982, 46, 1045-1061.

[35] W. H. E. Schwarz, E. WechselTrakokowski, Chem. Phys. Lett. 1982, 85, 94-97.

[36] R. H. Contreras, J. E. Peralta, Prog. Nucl. Magn. Reson. Spectrosc. 2000, 37, 321425.

[37] L. B. Krivdin, R. H. Contreras, Annu. Rep. NMR Spectrosc. 2007, 61, 133-245.

[38] K. Gholivand, Y. Maghsoud, M. Hosseini, M. Kahnouji, Magn. Reson. Chem. 2019, 57, S108-S116.

[39] V. Sychrovský, J. Šponer, L. Trantírek, B. Schneider, J. Am. Chem. Soc. 2006, 128, 6823-6828.

[40] V. Sychrovský, Z. Vokáčová, J. Šponer, N. Špačková, B. Schneider, J. Phys. Chem. 2006, 110, 22894-22902.

[41] Z. Vokáčová, M. Buděšínský, I. Rosenberg, B. Schneider, J. Sponer, V. Sychrovský, J. Phys. Chem. B 2009, 113, 1182-1191.

[42] L. Benda, Z. S. Vokáčová, M. Straka, V. Sychrovský, J. Phys. Chem. B 2012, 116, 3823-3833.

[43] L. Benda, B. Schneider, V. Sychrovský, J. Phys. Chem. A 2011, 115, 2385-2395.

[44] A. Vavřinská, J. Zelinka, J. Šebera, V. Sychrovský, R. Fiala, R. Boelens, V. Sklenáŕ, L. Trantírek, J. Biomol. NMR 2016, 64, 53-62.

[45] M. Pecul, M. Urbańczyk, A. Wodyński, M. Jaszuński, Magn. Reson. Chem. 2011, 49, 399-404. 